\title{
A IMPORTÂNCIA DOS OBJETOS DE APRENDIZAGEM NA EDUCAÇÃO AMBIENTAL E A SUA DISPONIBILIDADE NAS BASES DE DADOS RIVED E BIOE ${ }^{1}$
}

\author{
José Agripino de Araújo, UACEN/CFP/UFCG, \\ joseagripino1988@gmail.com
}

\begin{abstract}
RESUMO
$\mathrm{Na}$ atualidade o avanço tecnológico vem contribuindo para o crescimento $\mathrm{e} o$ desenvolvimento das ciências. O presente trabalho visa discutir os subsídios que as tecnologias podem trazer no intuito de melhorar a forma de trabalhar os conteúdos em sala de aula. A contribuição dos objetos de aprendizagem pode ser aliada com a interdisciplinaridade, e principalmente na atualidade, para o melhoramento da educação. Inserir objetos de aprendizagem para aprimorar o conhecimento sobre o meio ambiente pode contribuir para o aperfeiçoamento da aprendizagem dos alunos. Pensando na necessidade de aliar 0 conhecimento com a tecnologia, principalmente a capacidade de desenvolver novas formas de abordagens de conteúdo, relaciona-los com conteúdo da química ambiental, por exemplo, têm uma maior probabilidade de melhor a compreensão dos alunos. Na realização deste trabalho será desenvolvida uma pesquisa quantitativa a respeito do assunto: A importância dos Objetos de Aprendizagem (OAs) oferecidos em Base de Dados Nacionais e Internacionais para Educação Ambiental. Com isso temos que os OAs, podem auxiliar na aprendizagem e, ao mesmo tempo, integrar a sociedade às novas tecnologias, com abordagens de conteúdos que têm uma significância enorme para os alunos no tocante a sua formação.
\end{abstract}

PALAVRAS-CHAVE: Objetos de Aprendizagem; Novas Tecnologias na Educação; Química Ambiental.

\section{THE IMPORTANCE OF LEARNING OBJECTS OFFERED ON THE BASIS OF NATIONAL AND INTERNATIONAL DATA FOR ENVIRONMENTAL EDUCATION}

\begin{abstract}
Nowadays the technological advance has contributed a lot to the growth and development of the sciences, the present work aims to discuss the contributions that such technologies can

\footnotetext{
${ }^{1} \mathrm{O}$ presente trabalho (não) contou com apoio financeiro de nenhuma natureza para sua realização.
} 
bring in order to improve the way of working the contents in the classroom. The contribution of learning objects can be allied with interdisciplinarity, and especially nowadays, for the improvement of education, inserting the learning object for the better knowledge of the environment can contribute to an improvement of student learning. Thinking about the need to combine knowledge with technology, especially the ability to develop new forms of content approaches, relate them to the content of environmental chemistry for example, are more likely to succeed with regard to student understanding. In the accomplishment of this work a quantitative research will be developed regarding the subject: The importance of the OAs offered in National and International Database for Environmental Education with this we have that the OAs, have aided in learning and integrated the society with the new technologies, with Approaches that have a huge significance for students in their training.

KEY WORDS: learning objects; New technologies in Education; Environmental chemistry.

\section{LA IMPORTANCIA DE LOS OBJETOS DE APRENDIZAJE EN LA}

\section{EDUCACIÓN AMBIENTAL Y SU DESPONIBILIDAD EN LAS BASES DE}

\section{DATOS RIVED Y BIOE}

\section{RESUMEN}

En la actualidad el avance tecnológico viene contribuyendo para el crecimiento y el desarrollo de las ciencias. El presente trabajo trata de discutir los subsidios que las tecnologías pueden traer con el fin de mejorar la forma de trabajar los contenidos en el aula. La contribución de los objetos de aprendizaje puede ser aliada con la interdisciplinaridad, y principalmente en la actualidad, para el mejoramiento de la educación. La inserción de objetos de aprendizaje para mejorar el conocimiento sobre el medio ambiente puede contribuir al perfeccionamiento del aprendizaje de los alumnos. Pensando en la necesidad de aliar el conocimiento con la tecnología, principalmente la capacidad de desarrollar nuevas formas de abordajes de contenido, relacionarlos con contenido de la química ambiental, por ejemplo, tienen una mayor probabilidad de mejor comprensión de los alumnos. En la realización de este trabajo se desarrollará una investigación cuantitativa sobre el tema: La importancia de los Objetos de Aprendizaje (OA) ofrecidos en Base de Datos Nacionales e Internacionales para Educación Ambiental. Con eso tenemos que los OAs, pueden auxiliar en el aprendizaje y, al mismo tiempo, integrar la sociedad a las nuevas tecnologías, con enfoques de contenidos que tienen una significancia enorme para los alumnos en cuanto a su formación.

PALABRAS CLAVE: Objetos de Aprendizaje; Nuevas Tecnologías en la Educación; Química Ambiental.

\section{INTRODUÇÃO}

A inserção das tecnologias na educação, nos dias atuais, permite maior compreensão dos conteúdos, já que os jovens estão inseridos em um meio no qual estes possuem um 
domínio sobre elas e, com o seu uso, desenvolver uma educação que envolva os aspectos sobre ciências e meio ambiente é um passo determinante na qualidade do aprendizado destes. Os processos educacionais que envolve a aprendizagem com simulações e demonstração devem ser pensados no âmbito escolar, pois há um grande potencial para o desenvolvimento de novas ideias.

Segundo Tarouco (2003, p. 02)

Objetos educacionais (OEs), podem ser definidos como qualquer recurso, suplementar ao processo de aprendizagem, que pode ser reusado para apoiar a aprendizagem. O termo objeto educacional (learning object), geralmente aplica-se a materiais educacionais projetados e construídos em pequenos conjuntos com vistas a maximizar as situações de aprendizagem onde o recurso pode ser utilizado.

A rede mundial de computadores tem influenciado na forma como as pessoas se comunicam atualmente, e é uma importante ferramenta que vem interferindo significativamente a forma como as pessoas assimilam o conhecimento. Consequentemente, há também mudança nos moldes de educar essa nova geração, com o auxílio de recursos educacionais projetados para serem utilizados no ensino. Esse cenário tem levado a elaboração de pesquisas relacionadas à tecnologia educacional (TE), no sentido de desenvolver estudos de novas maneiras de empregar as tecnologias da informação e comunicação (TIC's) como ferramenta suporte no processo de ensino-aprendizagem, sobretudo em ambientes virtuais de aprendizagem (AVA).

Segundo Audino e Nascimento (2010, apud Miranda 2004 p. 14), a utilização sempre se refere a: "Quando nos referimos a objetos de aprendizagem, associamos a internet e ao uso dos computados recursos digitais dinâmicos, interativos e reutilizáveis em diferentes ambientes de aprendizagem elaborados a partir de uma base tecnológica”.

Um Objeto de Aprendizagem (OA) pode ser usado em diferentes contextos e em diferentes ambientes virtuais de aprendizagem (AVA), cada OA tem suas características próprias na sua parte visual, a qual faz o aprendiz interagir de forma mais autónoma com os conteúdos. A tecnologia também pode/deve ser caracterizada como ferramenta de ensino e mudança, e algumas vezes, quando incorporada de forma significativa, as inovações tecnológicas podem resultar em uma revolucionária quebra de paradigmas educacionais, consequentemente, a maior mudança poderá ser também o meio como os recursos educacionais serão projetados, desenvolvidos, integrados e analisados para serem utilizados e 
então disponibilizados para o ensino. Este trabalho tem a princípio o intuito de realizar um levantamento a respeito da importância da tecnologia na experimentação, aliando essa tecnologia ao desenvolvimento de técnicas para a construção do conhecimento cientifico dos alunos, com relação principalmente a questões ligadas diretamente ao meio ambiente.

\section{OBJETOS DE APRENDIZAGEM E O ENSINO DE QUÍMICA}

Segundo Melo (2011, p.6):

O computador é, hoje, um objeto que desperta grande interesse nas pessoas, principalmente no que se refere às crianças e adolescentes. Com isso, o uso de ferramentas digitais como apoio ao ensino pode passar a ser um tema de interesse geral no contexto da educação, como também um grande recurso para o ensino.

Partindo disto, os OAs tem uma característica importante, que é integrar de maneira mais fácil e didática o conhecimento com simulações práticas, visualizações de fenômenos químicos, entre outros, onde se procura mostrar aos alunos algumas experiências as quais ajudem ele a desenvolver uma aprendizagem significativa.

São necessários três principais requisitos para conceber a verdadeira aprendizagem significativa de maneira a construir o conhecimento lógico estruturado, o amadurecimento cognitivo a respeito do assunto que possibilite a sua conexão com o novo conhecimento; a necessidade de conecta-se o seu conhecimento com aquele que pretende-se absorver de maneira lógica (TAVARES, 2003, p. 56). Assim, os OAs são considerados grandes aliados para o desenvolvimento cognitivo dos alunos, proporcionando versatilidade às funções educativas do professor. Acrescentando ainda que tais ferramentas podem ser adaptadas para distintos ritmos e estilos de aprendizagem em seus mais diferentes níveis de conhecimento. Para Machado (2016, p. 106):

O emprego dos softweres educacionais (SE) permite demonstrar de forma muito mais real algum fenômeno em que envolve situações de analises de arranjos geométricos, que podem situações da química como: ligações químicas, arranjos atômicos, processos físico-químicos, e química orgânica também.

Nessa ideia o emprego desses OAs na educação têm um grande impacto na compreensão de assuntos que vão desde a matemática, passando pela física, química e 
também a área ambiental, como por exemplo: efeito estufa, chuvas ácidas, ciclo do carbono e etc. Machado diz (2016, p.104):

Assim, a química, entre outras ciências investigativas, também conclama para si uso e aplicação de tecnomídias específicas para promover a efetivação da aprendizagem científica. Tais ferramentas denotam sua potencialidade, reforçando a ação docente em sala de aula de modo a favorecer colaborativa e substancialmente a aprendizagem significativa dos conteúdos escolares.

A química envolve e faz parte de uma ciência investigativa, apresenta características próprias, diferenciando-se de outras ciências, levando em consideração conteúdos os quais é necessário a criatividade e a imaginação para que se tenha um entendimento destes, como, por exemplo, a explicação do comportamento dos átomos e moléculas. Os alunos que estão entrando em contato pela primeira vez com conhecimentos novos, não criam, a princípio, uma ideia do comportamento de tais espécies. Contudo o uso de simulações onde o aluno tem maior capacidade de desenvolver um conhecimento muito mais elevado, levando em consideração o amadurecimento intelectual dos alunos e suas capacidades cognitivas de aprendizagem.

\section{BANCOS DE DADOS DE OBJETOS EDUCACIONAIS}

As bases de dados que estão presente estes OAs são de fácil acesso, bastando apenas estar conectado com a internet, além disso, há opções de fazer download, para serem utilizados quando não existir disponível conexão, logo podendo ser utilizado na sala de aula sem maiores problemas. No presente trabalho procura-se enfatizar e evidenciar principalmente OAs quem estão presentes nas duas principais bases de dados a qual será estudada como a Rede Interativa Virtual de Educação (RIVED) e o Banco Internacional de Objetos Educacionais (BIOE). Segundo o sitio do RIVED: Em 1997 Brasil e Estados Unidos desenvolveram um acordo sobre o uso da tecnologia como ferramenta pedagógica. A parceria do Brasil em 1999 se deu por meio de duas Secretaria de governo, entre elas a Secretaria de Educação a Distância (SEED). Esta equipe foi responsável até o ano 2003 pela produção de 120 OA, que contemplavam as seguintes disciplinas: Biologia, Química, Física e Matemática para o Ensino Médio. Com a transferência em 2004 do processo de elaboração de objetos de aprendizagem para as universidades onde recebeu o nome de Fábrica Virtual. Com essa 
expansão houve a necessidade de contemplação e produção de novos conteúdos em outras áreas de conhecimento como ensino fundamental, profissionalizante e para atendimento às necessidades especiais.

O BIOE tem um total de [19.842] OAs publicados, sendo que [174] estão sendo avaliados ou aguardando autorização dos autores para a publicação, destes [10.289] estão relacionados diretamente com o ensino médio, divididos em suas respectivas grades curriculares. Este repositório está ligado diretamente ao ministério da educação MEC, e envolve parcerias com universidades do Brasil e de outros países.

\section{RESULTADOS E DISCUSSÕES}

\section{BANCO INTERNACIONAL DE OBJETOS DE EDUCACIONAIS (BIOE)}

No BIOE estão disponibilizados diretamente para a área da Química [1723] que se subdividem-se em Animações/Simulações [345], Áudios [195], Experimentos Práticos [295], Hipertextos [46], Imagens [311], Mapas [0], Softwares Educacionais [35], Vídeos [496]. Especificamente para a área da química ambiental existem em torno de [66] áudios, [6] experimentos práticos e [32] vídeos.

A figura 1 mostra o quanto o BIOE tem uma grande "elasticidade educacional" compreendendo desde de a educação infantil, ensino fundamental, ensino médio, ensino superior e modalidades de ensino, sendo que em todas estas categorias, existem suas subcategorias com seus respectivos assuntos sobre a modalidade escolhida.

Na modalidade Ensino Médio há cerca de [10.289], onde estes estão distribuídos em diferentes disciplinas, como mostra a Figura 2.

A figura 3 mostra um grande leque de escolhas como: Animações/Simulações, Áudios, Vídeos, Experimentos Práticos, Imagens, Mapas e etc. No contexto da química ambiental há em Animação/Simulações vários objetos educacionais, que podem ser integrados ao contexto da educacional ambiental. Existem ao todo [28] OAs, na qual a grande maioria destes são desenvolvidos por alunos ou professores brasileiros com parcerias de programas institucionais ou de universidades nacionais e internacionais. Nessa perspectiva, são detectados vários aspectos relacionados a esta temática no BIOE, onde as quantidades de 
OAs têm alcançado um número bastante elevado para os conteúdos que versem a temática ambiental e, principalmente, abrindo oportunidades no desenvolvimento de novas possibilidades de ensino-aprendizagem.

Figura 1: Tela de início do BIOE

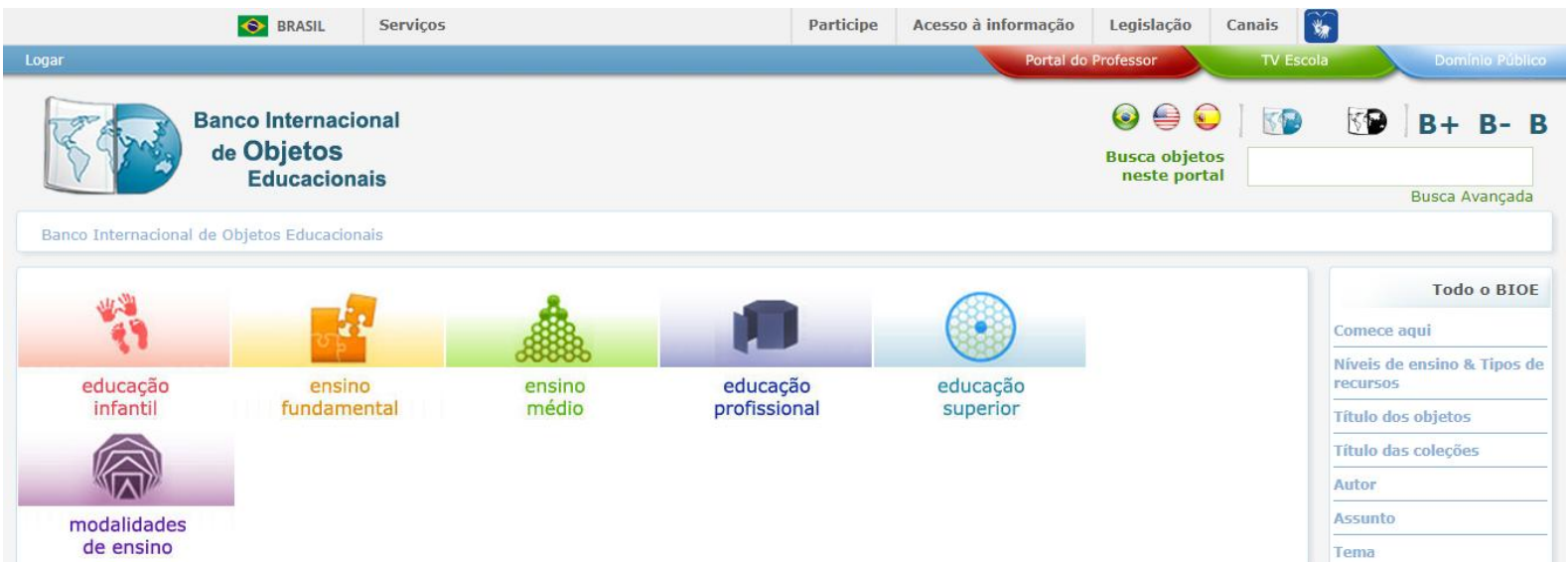

Fonte: BIOE. Acesso em: 18 de agosto de 2017.

Figura 2: Tela das disciplinas relacionadas com a base de dados

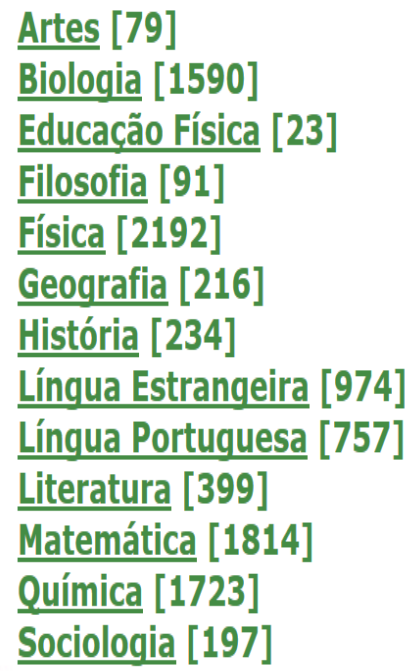

Artes [79]

Biologia [1590]

Educacão Física [23]

Filosofia [91]

Física [2192]

Geografia [216]

História [234]

Línqua Estrangeira [974]

Línqua Portuguesa [757]

Literatura [399]

Matemática [1814]

Química [1723]

Sociologia [197]

Fonte: BIOE. Acesso em: 18 de agosto de 2017. 
Figura 3: Tela das várias opções de objetos educacionais.

\begin{tabular}{|c|c|c|c|c|c|}
\hline जै & & $\$ 8088$ & & & $\sqrt{A}$ \\
\hline $\begin{array}{l}\text { educação } \\
\text { infantil }\end{array}$ & $\begin{array}{c}\text { ensino } \\
\text { fundamental }\end{array}$ & $\begin{array}{l}\text { ensino } \\
\text { médio }\end{array}$ & $\begin{array}{c}\text { educação } \\
\text { profissional }\end{array}$ & $\begin{array}{l}\text { educação } \\
\text { superior }\end{array}$ & $\begin{array}{c}\text { modalidades } \\
\text { de ensino }\end{array}$ \\
\hline Buscar em & & & & & Avançada \\
\hline Título & Autor & & & Assunto & Tema \\
\hline
\end{tabular}

Ensino Médio: Química: Animações/Simulações [345]

Ensino Médio: Química: Áudios [195]

Ensino Médio: Química: Experimentos Práticos [295]

Ensino Médio: Química: Hipertextos [46]

Ensino Médio: Química: Imagens [311]

Ensino Médio: Química: Mapas [0]

Ensino Médio: Química: Softwares Educacionais [35]

Ensino Médio: Química: Vídeos [496]

Fonte: BIOE. Acesso em: 18 de agosto de 2017.

\section{BASE DE DADOS RIVED}

O RIVED é um programa da Secretaria de Educação a Distância - SEED, que tem por objetivo a produção de conteúdos pedagógicos digitais, na forma de objetos de aprendizagem. Tais conteúdos primam por estimular o raciocínio e o pensamento crítico dos estudantes, associando o potencial da informática às novas abordagens pedagógicas. A meta que se pretende atingir disponibilizando esses conteúdos digitais é melhorar a aprendizagem das disciplinas da educação básica e a formação cidadã do aluno. Além de promover a produção e publicar na web os conteúdos digitais para acesso gratuito, o RIVED realiza capacitações sobre a metodologia para produzir e utilizar os objetos de aprendizagem nas instituições de ensino superior e na rede pública de ensino.

Matemática, Física, Biologia, Química e Língua Portuguesa, são alguns das disciplinas ofertadas nessa base de dados trazem relevantes contribuições para o ensino de conteúdos relacionados principalmente ao ensino médio, nessa base há também espaços para publicação de trabalhos científicos como artigos.

Na categoria ensino médio, na subcategoria Química, há OAs com o título Adubos, que tem o objetivo de identificar a presença da química na agricultura, classificando os principais adubos nitrogenados, fosforados e potássicos, conforme suas principais 
características, além de buscar conhecer os elementos químicos importantes para o metabolismo vegetal, como nutrientes e suas principais funções no vegetal.

Figura 4: Tela inicial do RIVED

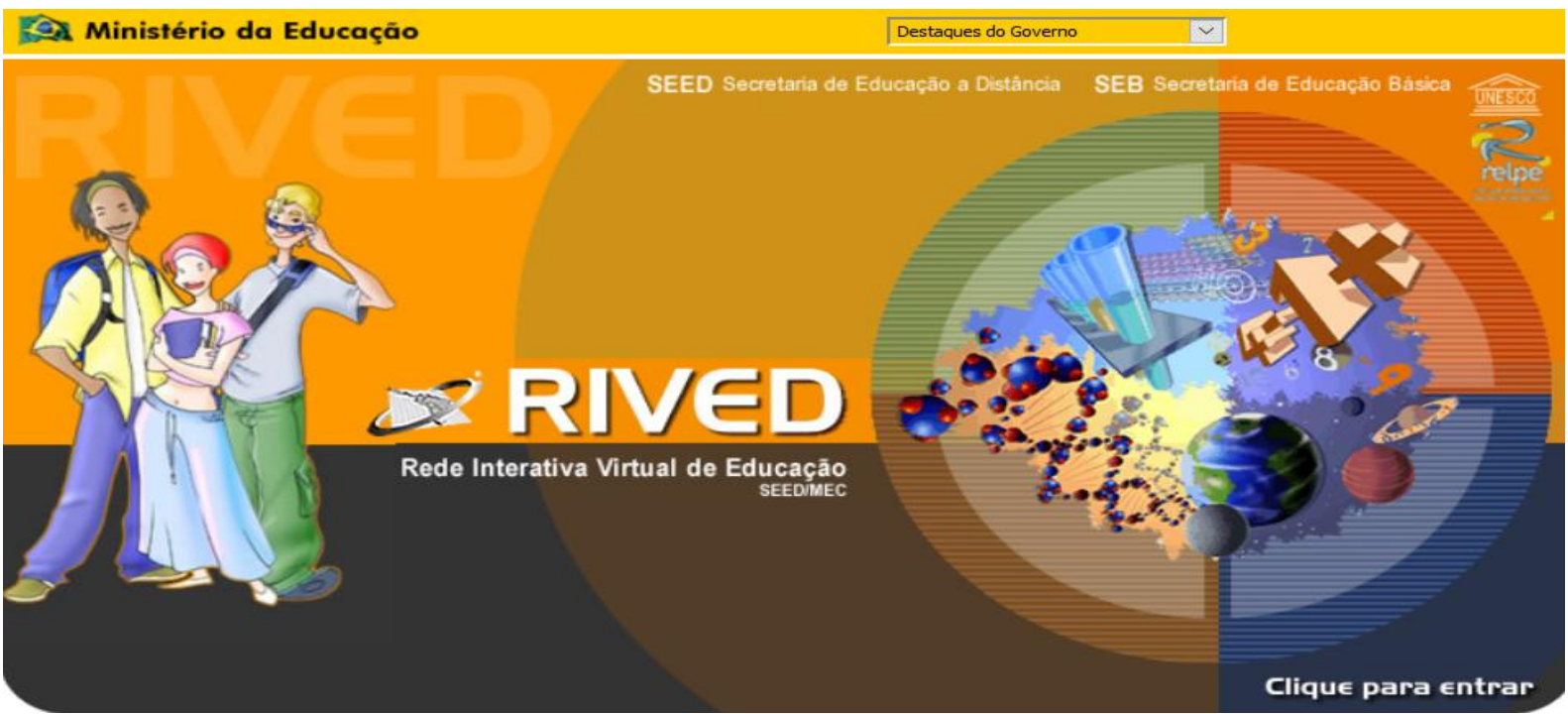

Fonte: RIVED. Acesso em: 18 de agosto de 2017.

Figura 5: Tela inicial por escolhas de assuntos de RIVED

\begin{tabular}{|l|}
\hline Conheça o Rived \\
\hline Fábrica Virtual \\
\hline Curso "Como usar" \\
\hline Como acessar \\
\hline Concurso Rived \\
\hline Artigos \\
\hline Informações Técnicas \\
\hline Padrões Rived \\
\hline Notícias \\
\hline Equipe \\
\hline Dúvidas \\
\hline Entre em contato \\
\hline Área Restrita \\
\hline
\end{tabular}

\section{Pesquisando no Rived...}

Pesquise atividades nas diversas áreas de conhecimento, tais como:

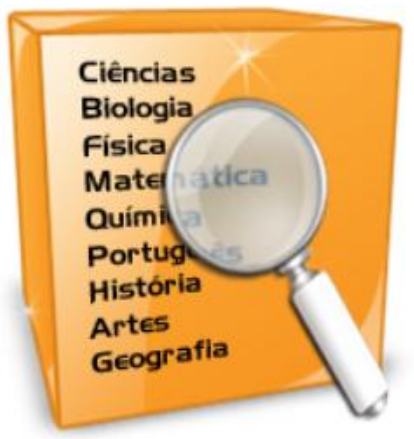

Para fazer a pesquisa digite uma área de conhecimento como as citadas acima ou uma palavra relacionada ao assunto de interesse. Ao digitar a área de conhecimento o sistema listará todos os conteúdos publicados nesta área. Para saber mais como fazer a busca, clique no menu ao lado, no botão "Como acessar".

Fonte: RIVED. Acesso em: 18 de agosto de 2017 
Com o AO da figura 6 o aluno pode desenvolver conceitos de ácido e bases de Arrenius, conhecer símbolos e relacionar com seu dia-dia, construir um maior conhecimento das causas e consequências das chuvas ácidas como mostra a Figura 6. Vemos o que é bastante comum nessas bases de dados, que é a abordagem no contexto da Química Ambiental, o efeito estufa, que é um problema recorrente dos conteúdos de química que envolve gases, com isso podemos demostrar de forma experimental tal efeito, suas causas e preocupação dos cientistas e da sociedade com o futuro do planeta.

Figura 6: Tela inicial do objeto de aprendizagem os mistérios químicos das chuvas acidas.

\begin{tabular}{|c|c|c|}
\hline$(4)$ & Tipo de 0bjetc & $\begin{array}{l}\text { Contédo produzido pala o } \\
\text { conculso Rived }\end{array}$ \\
\hline 4114 & I'tulo & $\begin{array}{l}\text { Os Mistérios oulmicos da Chuva } \\
\text { Acida }\end{array}$ \\
\hline 4 & Gérle & lasérie(Engino Médio) \\
\hline$y$ & Gategorld & Ouínica \\
\hline 0.211 & subcategolla & Acidos e oases \\
\hline
\end{tabular}

Fonte: RIVED. Acesso em: 18 de agosto de 2017

\section{CONCLUSÃO}

Podemos observar que os OAs, tem auxiliado na aprendizagem e integrado a sociedade a as novas tecnologias, com isso, se obtêm um melhor aprimoramento no tocante as abordagens de conteúdos tendo uma significância enorme para os alunos em formação. Estas novas tecnologias aliadas com um planejamento adequado dos conteúdos a serem abordados têm uma grande chance de se conseguir atingir os objetivos antes planejados e, 
consequentemente, os alunos internalizarem o conhecimento com maior facilidade e, por conseguinte, construindo uma aprendizagem significativa.

Com relação ao número de objetos disponíveis, o BIOE é o principal banco de dados, tendo uma navegação mais fácil e oferecendo uma maior quantidade de OAs relacionados ao tema Ambiental quanto comparado ao RIVED, onde este dispõe pouco material na área de química ambiental.

Frente aos grandes problemas causados pelo homem a natureza este assunto deveria ter um foco muito maior, pois produzir conhecimentos que levem a conservação do planeta é imprescindível, já que este é a casa de milhares de espécies existentes.

\section{REFERENCIAL TEÓRICO}

MACHADO, A. S. Uso de Softwares Educacionais, Objetos de Aprendizagem e Simulações no Ensino de Química. Química nova na escola, Vol. 38, N$^{\circ}$ 2, p. 104-111, 2016. Disponível em: http://qnesc.sbq.org.br/online/qnesc38_2/03-QS-76-14.pdf. Acessado em: julho 2017.

TAVARES, R. Aprendizagem significativa em um ambiente multimídia. In: V Encuentro Internacional sobre Aprendizaje Significativo 11 a 15 set/2006 - Madrid - Espanha. Disponível em: <http://rived.mec.gov.br/artigos/2006-VEIAS.pdf>. Acessado em: 13 julho 2017.

TAROUCO, L. M. R.; FABRE, M.C.J.M.; TAMUSIUNAS, F.R. Reusabilidade de objetos educacionais. Revista Renote Novas Tecnologia na Educação, v. 1, № 1, Fevereiro, 2003. Disponível em: <http://seer.ufrgs.br/renote/article/view/13628>. Acessado em: 09 junho 2017.

MELO, D. M. B. Jogos digitais e objetos de aprendizagem no ensino da matemática. In: III Encontro Regional em Educação Matemática (SBEM/RN) Disponível em: <www.pucrs.br/famat/viali/tic_literatura/artigos/objetos/CC_Melo_e_Silva.pdf >. Acessado em: 20 julho 2017.

AUDINO, D. F.; NACIMENTO, R. S. Objeto de Aprendizagem - Diálogos Entre Conceitos e Uma Nova Proposição Aplicada à Educação. Revista Contemporânea de Educação, vol. 5, n. 10, jul/dez 2010. Disponível em: 〈https://revistas.ufrj.br/index.php/rce/article/view/1620>. Acessado em: 29 julho 2017. 
SILVA, E. K. S. et al. Caracterização dos Objetos de Aprendizagem para o Ensino Médio de Química. In: XIV Congresso Internacional de Tecnologia na Educação, Brasil, Recife, Setembro de 2016.

Disponível

em:

<http://demo.cubo9.com.br/senac/pdf/poster/016.pdf〉. Acessado em: 04 Agosto 2017. 